\title{
Energy Trapping Effect of Stepped Electrode in Miniature AT-Cut Quartz Resonator
}

\author{
S.Y. Pao, Robin Huang, and C.S. Lam \\ TXC Corporation, Ping Cheng City, Taoyuan County, Taiwan
}

\begin{abstract}
As the size of quartz resonator continues to shrink, beveling of the plano-plano quartz crystal is needed to reduce mounting loss and to sustain energy trapping so to have low motional resistance for oscillation. The beveling process though is time consuming and difficult to control for small size and low frequency quartz crystals. In recent years, studies on stepped electrode (a.k.a bi-mesa electrode) began to appear. It's believed that stepped electrode on plano-plano crystal would do part of the job in energy trapping like beveled crystals. In this paper, we report our own study of stepped electrode with the following configurationssingle layer electrode on plano-plano crystal, stepped electrode on plano-plano crystal, single layer electrode on beveled crystal, and stepped electrode on beveled crystal. The vehicle for this study was a $15 \mathrm{MHz} 3.2 \mathrm{mmx} 2.5 \mathrm{~mm}$ crystal. Preliminary results show that stepped electrode can provide to some degree of energy trapping as bevel crystals.
\end{abstract}

\section{INTRODUCTION}

As the demand of personal communication, the wireless apparatus, like cell phone and global positioning system (GPS), become smaller and lighter, size and power consumption are the main concern for the components in miniaturized portable electrical device. To answer this trend, the heart of electrical circuit- quartz crystal resonator/oscillator, is now reduced its size from $7 \mathrm{mmx} 5 \mathrm{~mm}$ to $2 \mathrm{mmx} 1.6 \mathrm{~mm}$, and will be shrunk further. However, the size reduction also sacrifices the resonator's crystal impedance and Q-value. Trapping the thickness shear vibration energy of AT-cut quartz plate can keep the performance of a low frequency or thicker resonator. The most common way to trap the vibration energy is to bevel the plano-plano quartz plate to a convex-plano or convex-convex plate. The analysis and simulation of the energy trapping effect has been studied by Tanaka et al [1, 2]. Another method to trap the energy and decouple the unwanted modes is by stepped electrode [3, 4].

In this paper, we adopt both the stepped electrode and beveling methods to trap the vibrational energy in the central area under the electrode of the blank for keeping low crystal impedance and high Q-values. We set four group of blanks with different electrodes and beveling conditions to verify the effect of stepped electrode. These experiments are based on the blank for $3.2 \mathrm{mmx} 2.5 \mathrm{~mm}$ size and $15 \mathrm{MHz}$ frequency. The experimental results show that a proper stepped electrode design can achieve good energy trapping effect.

\section{ENERGY TRAPPING OF AN INFINITE AT-CUT QUARTZ PLATE}

The energy trapping theory is based on the standing wave principle that resonances can only occur at frequencies above the cut-off frequency [5]. As we consider an infinite AT-cut plate having a thickness $t$, its fundament thickness shear resonant frequency is 


$$
f_{B}=\frac{1}{2 t}\left(\frac{c_{66}^{\prime}}{\rho}\right)^{1 / 2}
$$

where $c_{66}^{\prime}$ is the stiffness constant of AT-cut quartz plate, $\rho$ is the density of quartz. If we plate electrode on the quartz plate in designated area $\mathrm{A}$ surrounded by unplated area $B$, the mass loading effect of electrode makes the effective thickness of region $\mathrm{A}$ equal to $t^{\prime}$. The frequency of the electrode region is

$$
f_{E}=\frac{1}{2 t^{\prime}}\left(\frac{c_{66}^{\prime}}{\rho}\right)^{1 / 2}
$$

The cut-off frequency of electrode region $\mathrm{A}$ and unplated area $\mathrm{B}$ are $f_{E}$ and $f_{B}$ respectively. Waves having frequencies higher than $f_{E}$ and lower than $f_{B}$ can exist in region A only because frequencies lower than $f_{B}$ can not exit the unplated area.

Waves in region A having frequencies between $f_{E}$ and $f_{B}$ are totally reflected to become standing waves. If these waves are excited by the correct frequencies, there will become spurs. Waves having frequencies higher than $f_{B}$ are traveling outward toward the unplated region and not reflected to form standing waves. In order to suppress all inharmonic overtone modes in a resonator with circular electrodes, we have [5]

$$
\text { Plate-back }<f_{B} \frac{2.98}{n^{2}}\left(\frac{t}{d}\right)^{2}
$$

where $\mathrm{n}$ is the overtone number, $\mathrm{d}$ is the diameter of the circular electrode.

\section{EXPERIMENT RESULTS}

The sketch of beveled and stepped electrode quartz crystal plates are shown in Figures 1 and 2.

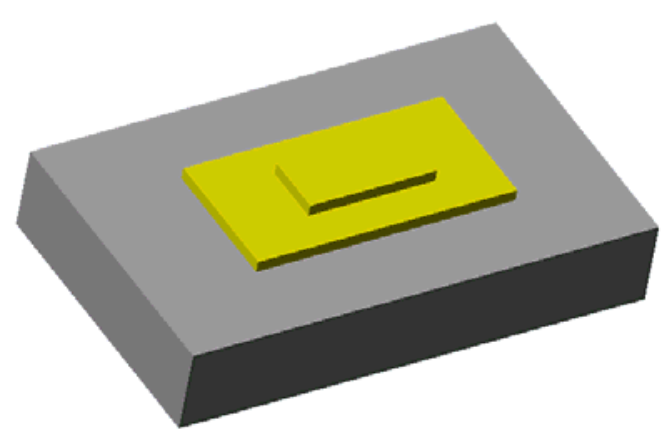

Figure 1. The sketch of a stepped electrode quartz plate

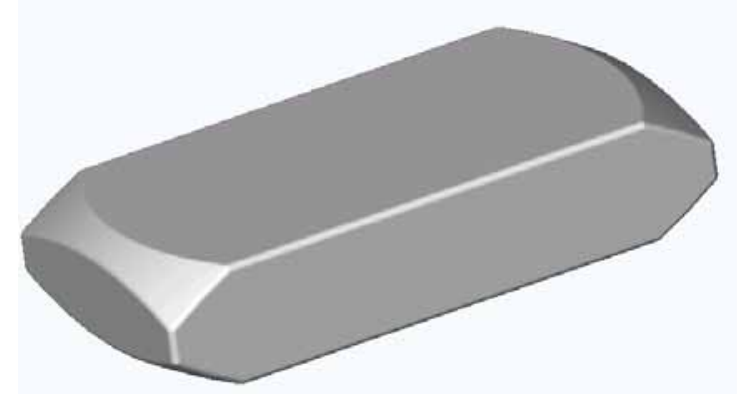

Figure 2. The sketch of a beveled quartz plate

We set four group chips with different stepped electrodes and beveling condition to verify the effect of stepped electrode. $\mathrm{T}$ he vehicle of this study are all $3.2 \mathrm{mmx} 2.5 \mathrm{~mm}$ size $15 \mathrm{MHz}$ resonator. The conditions of each group are listed in Table 1.

\begin{tabular}{|c|c|c|c|}
\hline Group & $\begin{array}{c}\text { Electrode } \\
1.19 * 2.28 \mathrm{~mm}^{2}\end{array}$ & $\begin{array}{c}\text { Electrode } \\
0.8 * 1.65 \mathrm{~mm}^{2}\end{array}$ & $\begin{array}{c}\text { Proper } \\
\text { Bevel }\end{array}$ \\
\hline$\# 1$ & $\sqrt{ }$ & $\times$ & $\times$ \\
\hline$\# 2$ & $\sqrt{ }$ & $\sqrt{ }$ & $\times$ \\
\hline$\# 3$ & $\sqrt{ }$ & $\times$ & $\sqrt{ }$ \\
\hline$\# 4$ & $\sqrt{ }$ & $\sqrt{ }$ & $\sqrt{ }$ \\
\hline
\end{tabular}

Table 1. The stepped electrode and beveling condition of each group

The blank in the first group is plated one metal electrode layer $\left(1.19 * 2.28 \mathrm{~mm}^{2}\right)$ but without bevel. The blank in the second group is plated two metal electrode layers $\left(1.19 * 2.28 \mathrm{~mm}^{2}\right.$ and $\left.0.8 * 1.65 \mathrm{~mm}^{2}\right)$ but without bevel. The blank in the third group is plated one metal electrode layer $\left(1.19 * 2.28 \mathrm{~mm}^{2}\right)$ and with proper bevel. The blank in the last group 
is plated two metal electrode layers $\left(1.19 * 2.28 \mathrm{~mm}^{2}\right.$ and $0.8 * 1.65 \mathrm{~mm}^{2}$ ) and with proper bevel. We use the equivalent series resistance (ESR) of the quartz resonator in each group as the index of energy trapping efficiency. The ESR (eliminating outliners) of each group are listed in T able 2.

\begin{tabular}{|c|c|c|c|c|c|}
\hline \multicolumn{2}{|c|}{ Group } & $\# 1$ & $\# 2$ & $\# 3$ & $\# 4$ \\
\hline ESR & Ave. & 142.8 & 39.5 & 6.7 & 5.9 \\
\cline { 2 - 6 }$($ Ohm) & Stdev. & 46.1 & 11.1 & 1.8 & 1.4 \\
\hline
\end{tabular}

Table 2. The average and standard deviation of ESR of each group

The statistic analyses by MINITAB of the ESR of each group are in figure 3 to 6 .

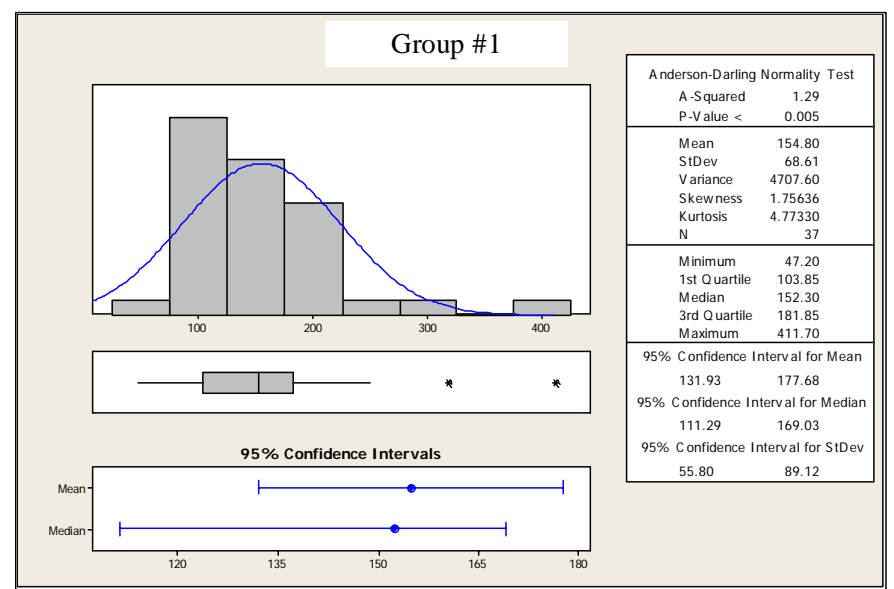

Figure 3. The ESR statistic analysis of group \#1

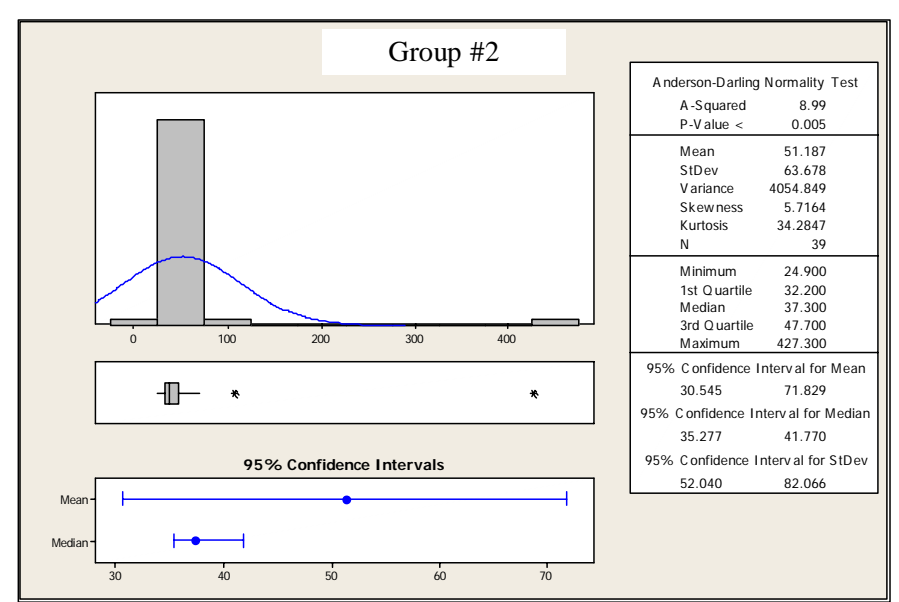

Figure 4. The ESR statistic analysis of group \#2

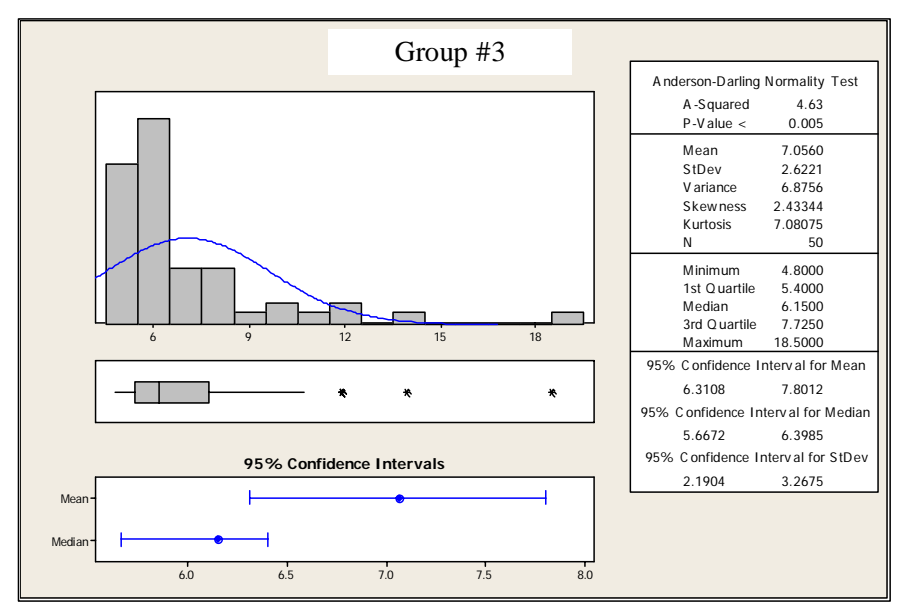

Figure 5. The ESR statistic analysis of group \#3

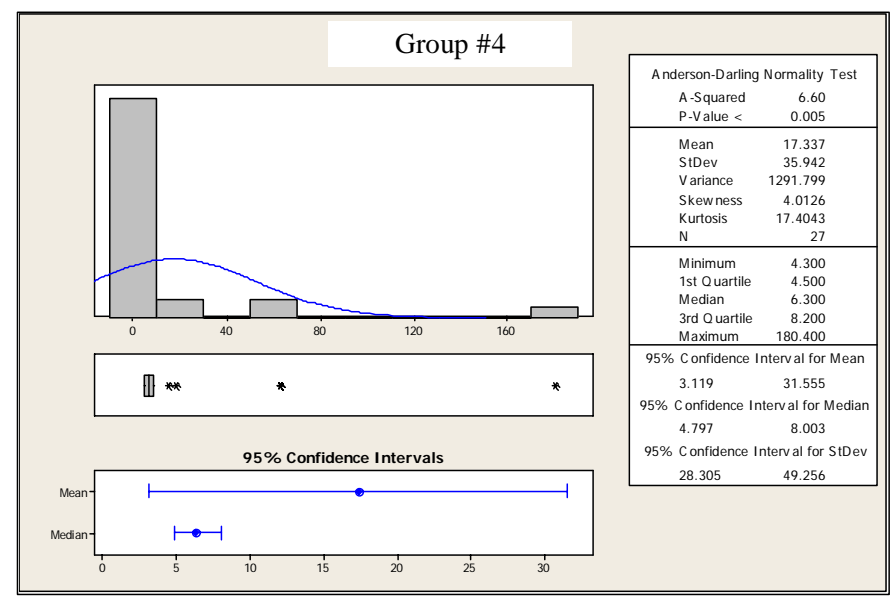

Figure 6. The ESR statistic analysis of group \#4

The experimental results show that blank with only one electrode but without proper bevel (group \#1) has the highest ESR. That means the chips in group \#1 can trap only a small amount of vibrational energy. The blank with two stepped metal electrodes but no bevel (group \#2) has lower ESR which is only $28 \%$ of that of group $\# 1$. The ESR of group \#3 (blank with proper bevel and only one electrode layer) is only $5 \%$ of that of group \#1. Group \#4 tells us that as we apply the stepped electrode on a beveled quartz plate, we can lower the ESR further. Because the ESR of quartz plate with proper bevel is already very low, stepped electrode can only enhance the impedance characteristic a few more. However, if we compare the ESR standard deviation and distribution of group \#3 with that of group \#4, we can find that 
stepped electrode makes ESR characteristic more concentrate, and it is good for controlling the quality of resonators.

\section{CONCLUSION}

For low frequency or thick quartz plate, trapping the vibrational energy for high Q-value and low crystal impedance is necessary. Beveling is a very effective way to achieve this purpose. However, in miniature resonator, to bevel the quartz plate is time consuming and difficult to control. By the experiment results in this study, applying stepped electrode on a quartz plate without bevel can lower the ESR obviously. Even for beveled crystal, stepped electrode can also concentrate the ESR characteristics and let the quality be more stable. With proper design in the number of layers, area and thickness of each electrode, the crystal impedance can be lowered more to fit the demand of oscillator circuit. In miniature quartz resonator, stepped electrode is a good choice to do the job of energy trapping either with beveling or independently.

\section{REFERENCES}

[1] H. Sekimoto, "Analysis of Trapping energy Resonators with Circular Electrodes," IEEE Transactions on Sonics and Ultrasonics, Vol. SU-31, No. 6, Nov. 1984

[2] M. Tanaka, T. Imai, and Y.K. Yong , “3-D FEM Einenvalue Analysis of Relative Impedance and Energy Trapping of Resonant Modes in AT-cut Resonators,” 2003 IEEE Freq. Control. Symp, pp. 709-714

[3] S. Goka, K. Tamura, H. Sekimoto, Y. Watanabe, and T. Sato, "Decoupling Effect of MultiStepped Bi-mesa AT-cut Quartz Resonators" 2003 IEEE Freq. Control. Symp, pp. 694-697

[4] F. Lu, H.P. Lee, and S.P. Lim, “EnergyTrapping Analysis for the Bi-stepped Mesa Quartz Crystal Microbalance Using the Finite Element Method," Smart Mater. Struct. 14, pp. 272-280, 2005

[5] V.E. Bottom, Introduction to Quartz Crystal Unit Design, VNR, pp.168-177 